\title{
Exploring Emotional ‘Dialogues’ in Spontaneous Twin Infant-Mother Interaction
}

\author{
Maria Markodimitraki ${ }^{1}$ and Theano Kokkinaki ${ }^{*}$ \\ ${ }^{1}$ Department of Preschool Education, University of Crete, Rethymnon, 74100 Gallos, Greece \\ ${ }^{2}$ Department of Psychology, University of Crete, Rethymnon, 74100 Gallos, Greece
}

\begin{abstract}
Background: This study investigated emotional coordination and mismatching in spontaneous twin infant-mother interaction.

Methods: Six twin infant-mother dyads were video-recorded during naturalistic interactions from the 2nd to the 6th month.

Results: a) Emotional coordination (matching of the type of facial expression of emotion, completement of the positive valence of facial expression of emotion and attunement in the shifts of emotional intensity direction) along with mismatching of the type of facial expression of emotion takes place in twin infantmother interactions. Emotional matching and mismatching occur at similar rates, b) Infants cause mismatching more than mothers do; and c) Infant age did not affect the developmental trajectories of emotional coordination and mismatching.

Conclusion: This study extends empirically the theory of innate intersubjectivity to twin infant-mother interaction and provided evidence that mothers and twin infants adjust the timing, form and energy of their emotions to obtain inter-subjective coordination and inter-motive adjustments.
\end{abstract}

\section{Publication History:}

Received: June 28, 2014

Accepted: October 25, 2014

Published: January 04, 2015

\section{Keywords:}

Emotional coordination, Emotional matching, Emotional mismatching, Emotional completement, Emotional attunement, Twins, Innate intersubjectivity

\section{Introduction}

We investigated the emotional coordination and mismatching in spontaneous twin infant-mother interactions. The evidence of this study may extend our understanding on the way in which being a twin infant in a particular type of interaction with the twin mother in the context of twin-ship, may influence the ways in which these children come to form significant interpersonal connections.

The temporal organization of dyadic emotional states in face-to-face parent - infant interaction has been described through the application of various terms which imply differences in conceptualizing how infants express their understanding by engaging in reciprocal responses to the adult's social signals [1]. The convergent point of these mechanisms is that infants express their ability of direct perception of feelings and interests in another person and responsive attunement manifested through emotionally regulated engagements [2]. However, little attention has been paid to the evidence that interaction frequently moves from coordinated states to less or miscoordinated states. This is a paradox given that more than $70 \%$ of the time of mother-infant interaction is spent in mismatching states, and the occurrence of coordination varies from not at all to $30 \%-40 \%$ of the interaction time $[3,4]$. Miscoordinated states have been attributed to interactive errors $[4,5]$, and transitions from coordinated to less coordinated states have been attributed to a process of repair $[4,6]$, or to the maintainance of some optimal level of excitement or arousal [7].

A number of classic twin studies and a restricted number of twin-family studies has investigated certain behavioral measures conceptually related to emotional coordination such as empathy, synchrony, emotion regulation, theory-of-mind abilities, affect attunement and social structure [8-14]. The results of these studies remain contradictory and provide evidence of differential developmental pattern of affective measures in the context of the complex interplay of heredity and environment in the origins and maintenance of concern for other. In particular, the comparison of singleton- and twin infant-mother interactions showed that at 3 and 6 months, mothers of singletons scored higher than mothers of twins in maternal sensitivity, or there were no differences in the levels of maternal sensitivity between singletons and twins at 6 months.
With no intention to underestimate the value of these studies, they lack the systematic investigation of interactional, self-other regulation dynamics of spontaneous twin infant-mother interaction. Further, in twin-family studies: a) within long intervals between video-recordings; b) emotional regulation focused on individual-specific expressed emotions $[11,14]$; c) emphasis on the study of emotional regulation was paid rather on maternal than infant expressed emotions, while indifference/unresposiveness has been studied exclusively on twin infant side as early as 14 months; d) child initiation of interaction bids has been studied as early as 6 months. Affective measurement of child-led interactions was restricted to child positive affect; and e) the coder analyzed selected portions of an interaction $[11,13]$.

We aim to investigate emotional coordination and mismatching taking place in twin infant-mother spontaneous interaction during early infancy. We aim to advance the methodology of the previous studies because: a) micro-analysis (in accuracy of $1 / 25^{\text {th }}$ of a second) of twin infants' and maternal facial expressions of emotion will be continuous; and b) longitudinal studies that obtain data at a sufficient frequency to track age-related changes are relatively rare [15] and relationships emerging between parents and each of the multiple infants have rarely been examined longitudinally [11]. However, we recognize methodological issues. This study is based on micro-analysis of facial expressions of emotion of a restricted sample of 6 twin infant-mother pairs, each of which was video-recorded for 7 minutes from the $2^{\text {nd }}$ to the $6^{\text {th }}$ month of infants' life at 30 -day intervals. One has to take into consideration that microanalysis of non-verbal expressive behaviors is

"Corresponding Author: Dr. Theano Kokkinaki, Department of Psychology, University of Crete, Rethymnon, 74100 Gallos, Greece, Tel:++30-8310-77536; Fax: ++30-8310-77578; E-mail: kokkinaki@uoc.gr

Citation: Markodimitraki M, Kokkinaki T (2015) Exploring Emotional 'Dialogues' in Spontaneous Twin Infant-mother Interaction. Int J Psychol Behav Anal 1: 101 doi: http://dx.doi.org/10.15344/2455-3867/2015/101

Copyright: ( 2015 Markodimitraki et al. This is an open-access article distributed under the terms of the Creative Commons Attribution License, which permits unrestricted use, distribution, and reproduction in any medium, provided the original author and source are credited. 
Citation: Markodimitraki M, Kokkinaki T (2015) Exploring Emotional `Dialogues' in Spontaneous Twin Infant-mother Interaction. Int J Psychol Behav Anal 1: 101. doi: http://dx.doi.org/10.15344/2455-3867/2015/101

a difficult and time-consuming enterprise [16], and this is doubly true when the sample is as difficult to obtain as ours (twins) [17]. Further, one has to take into account the additional work required to collect data in Heraklion, the capital of Crete (the largest island of Greece). In connection to this, Legg and Roberts (1997) note that one cultural element that seems antithetical to modernization in the prefecture of Heraklion in Crete is the distrust of outsiders (such as the researchers in this case) and reliance on the family for protection [18]. This has to be added to the difficulty in assessing larger number of comparable families with healthy infants for an extensive observational period [19].

This study has been carried out within the frame of Trevarthen's theory of innate intersubjectivity [20-24]. According to this theory, infants possess functions of intersubjectivity, that is, they possess motives (which take recognizable form as emotions) that are specifically adapted to perceive, respond to, attract and influence how other persons feel and what they, in response, will perceive and do. This readiness for intersubjective work is reflected on the state of emotional equilibrium within the self (intra-subjective coherence). Intersubjective encounters with infants reveal coordination within - and between - subjects (Significant Others and infants) in three essential dimensions of communication that motivate learning in a human community: "kinematics" (timing), "physiognomics" (forms) and "energetics" (intensity) [24]. Emotional narratives constitute the fundamental carriers of information about motivational changes in the short term. The close following of emotions between persons in direct and intimate communication permits transfer and imitation of internal motives by which each is regulating the dynamics of consciousness and purpose [21,22]. Clear cycles of attention, anticipation (as a result of a sympathetic regulation of arousal, via communication of emotion states), and change in emotional arousal and in exchanged expressions of enjoyment have been described in interaction of a 11-month old twin girl with her father [15]. Further, Markodimitraki's case study [25] provided evidence of similar transient emotional expressions during spontaneous imitation in dyadic twin infant-mother interactions ( $1^{\text {st }}$ to $10^{\text {th }}$ month of infants life).

Studying emotional coordination along with mismatching in twin infant-mother spontaneous interaction during early infancy is important because assessments that focus on coordination are likely to see interactive failure, or pathology when neither is present [4] In connection to this, one has to take into consideration that the interactional environment of twin infants constitutes of unique and different features compared to singletons'. Mothers of multiple infants complain of having no energy to form a unique emotional bond with each child and report emotional distancing from their infants [11]. On one hand, twinship provides the unique experience of being a mirror image of one another, an identical in appearance to oneself, an infant of the same age $[13,26]$. In this context, the "other", the "like" mind in question, is a mind on whose workings they have uniquely abundant opportunities to reflect [8]. On the other hand, general developmental lags as well as more specific lags in self-other differentiation have been assumed for twins [13].

Emotional coordination was measured with three measures: a) matching (one partner expressed the type of facial expression of emotion of the other partner), b) completement [one partner expressed the positive valence of facial expression of emotion (pleasure, interest) of the other partner]; and c) attunement (one partner expressed the shifts in the direction of emotional intensity of the other partner). Emotional mismatching was coded when one or either partner was not interested in interacting with the other partner. We addressed the following hypotheses: (1a): Emotional coordination (matching, completement and attunement) along with mismatching will be evidenced in twin infant-mother interactions; (1b) The occurrence of mismatching will predominate over the occurrence of matching and completement; (2) Infants will direct emotional coordination and mismatching more than mothers do; (3) The facial expressions of emotion of each partner will remain stable when emotional expressions are compared between the beginning and the end of infant-directed speech; (4): Infant age will affect the developmental course of emotional coordination and mismatching across the age range of this study. Given: a) that exposure to emotional coordination may have a lasting impact on the infant's brain, particularly during the early plastic period of brain maturation; and b) the role of coordination in sensitizing infants to the emotional resonance that underlies human relationships across the life span and in shaping the adolescent's capacity for empathy [27], we assume that the early interactional environment of twin infants, which may be featured by limited exposure to coordination and high occurrence of mismatching, may affect the way in which twins form significant interpersonal relationships.

\section{Method}

\section{Participants}

Participants were part of a longitudinal and naturalistic study which aimed to compare basic aspects of spontaneous imitation in twin-infant- and singleton-mother interactions (see Funding). Six Greek, Cretan mother-twin infant pairs took part in this study (N $=6$ twin infants and six mothers $=12$ ). This study was restricted to the inclusion of first-born twin infants due to differences in the brain structure between first-born and second-born twins [28]. All infants in the study were healthy and born without complications, they came from families in which at least one parent was employed and the mothers were $>20$ years of age. No twins who were in medical risk or with birth weight less than 1,700 gr or with gestational age less than 34 weeks were selected. Table 1 shows demographic information for the sample of this study (Table 1).

\section{Twin zygosity determination}

Opposite-sex twin infant pairs were classified as dizygotic on the basis of the sex difference [30]. For same-sex twin infant pairs, zygosity was established according to the Zygosity Questionnaire for Young Twins [31].

\section{Procedure}

After parents approved their participation in the study [see 32 for the recruitment procedure and recording arrangements], an introductory discussion took place at their home, and the first visit for recording was arranged. Video-recordings of spontaneous mother-twin infant interactions at infants' home were made at 30-day intervals, from the beginning of the Primary Intersubjectivity Period (2 to 4.5 months) until the middle of the Period of Games (4.5 to 6 months). These periods refer to the coordination of self and other in interaction based on correspondences of timing, form, and intensity [21].

Each recording lasted 7 minutes. In the course of this study, five video-recordings were made for each twin-infant-mother pair, giving a total of 30 video-recordings for the entire sample (6 infants X 5 age 
Citation: Markodimitraki M, Kokkinaki T (2015) Exploring Emotional `Dialogues' in Spontaneous Twin Infant-mother Interaction. Int J Psychol Behav Anal 1: 101. doi: http://dx.doi.org/10.15344/2455-3867/2015/101

\begin{tabular}{|l|l|l|l|}
\hline & Mean & \multicolumn{1}{|c|}{ SD } & Range \\
\hline Mother Age (years) & 29.83 & 4.49 & $26-36$ \\
\hline $\begin{array}{l}\text { Mother Education } \\
\text { (years) }\end{array}$ & 13.66 & 4.80 & $6-20$ \\
\hline Father Age (years) & 34.66 & 5.60 & $26-40$ \\
\hline Father Education (years) & 12.0 & 5.05 & $6-18$ \\
\hline Gestational Age (weeks) & 35.3 & 1.21 & $34-36$ \\
\hline Birth Weight (kg) & 2,455 & 254.06 & $\begin{array}{l}2,100- \\
2,800\end{array}$ \\
\hline Birth Height (cm) & 49 & 1.16 & $48-51$ \\
\hline Breastfeeding (days) & 56.25 & 33.36 & $\begin{array}{l}30-105 \\
\text { (for four twin } \\
\text { infants) }\end{array}$ \\
\hline Sex of twin pairs & $\begin{array}{l}\text { two same-sex and four opposite- } \\
\text { sex pairs }\end{array}$ \\
\hline Male/ Female & $66.6 \%$ (male) \\
\hline Way of Delivery & Casaerian Section \\
\hline Birth order & \multicolumn{2}{|l|}{ First born children in the family } \\
\hline Family Compostion & Two-parent families \\
\hline Socio-economic Stastus & Middle-class families [32] \\
\hline
\end{tabular}

Table 1: Demographic Information for the Sample $(\mathrm{N}=6$ twin infant-mother pairs).

points). The data collection predicted 210 minutes of recordings (6 infants X 5 age points X 7 minutes). However, at the end of the study, we finally had 203 minutes of recordings. This data loss was due to one visit that was missed at 6 months due to short-term health problems of one twin infant pair. All recordings were made with a Handy Cam SONY DCR-HC90E Pal WideLCD.

The only instruction given to the mothers was: "Play as you normally do with your baby". The recordings took place in a room and a position chosen by the mother where there would be no interruption by a third-party.

\section{Coding}

Within well-defined units and subunits of analysis, micro-analysis of twin infant and maternal facial expressions of emotions was continuous (in accuracy of $1 / 25^{\text {th }}$ of a second), since the onset time in the expression of an emotion of one partner was also the offset time of the previous emotion of the same partner. Microanalysis of maternal and infant facial expressions of emotions was carried out according to six types of facial expressions (pleasure to the partner, interest to the partner, neutral expression, pleasure to the inanimate world, interest to the inanimate world and negative expression), three qualities of valence (positive, neutral and negative), and four categories for the direction of intensity change (ascending, descending, stable and fluctuating)

Within each sub-unit of analysis, interpersonal engagement was coded according to the: (a) type of facial expressions of emotion (matching, mismatching); and (b) valence of facial expressions of emotion (attunement, completement). Emotional matching was coded when one partner expressed the type of facial expression of the other other partner, i.e. both partners expressed pleasure to each other. Emotional mismatching was coded when one or either partner was not interested in interacting with the other partner. For example, one partner expressed interest or pleasure to the other partner, while the other was neutral or negative in emotion, or one partner was neutral in emotion while the other was negative. Each of these categories was coded for the whole duration of the subunit - in instances of both partners' stable emotional expressions - or at one time point of sub-unit of analysis when the emotional expressions of both partners were nonstable. Interpersonal engagement categories according to the valence of facial expression of emotion were coded as emotional attunement and completement. Before defining attunement and completement it is noted that, in the course of one subunit of analysis, it was most likely for each partner to express more than one emotional state. In order to obtain a description of the change of intensity over time, each facial expression within each category of emotional valence represented a symbol in the following scale: (a) positive emotional valence consisted of pleasure directed to the partner $(+++)$, pleasure directed to the inanimate world $(++)$, and interest directed to the partner $(+),(b)$ neutral emotional valence consisted of the neutral facial expression and the interest directed to the external world (0), and (c) negative emotional valence consisted of the negative facial expression (-). The sequence of the above symbols of each partner in the course of each subunit of maternal infant-directed speech determined four categories for the direction of intensity change: ascending, descending, stable and fluctuating. Emotional attunement was identified when one partner matched the shifts of emotional valence of the other partner (e.g. in ascending attunement, the valence of both partners' emotional state at the end of parental speech was higher in the scale than the valence of their emotional states in the beginning of parental speech, e.g. when both partners changed from interest directed to the partner $(+)$ to pleasure $(+++)$ directed to the partner]. It has to be noted that emotional matching and attunement are different measures. Matching focuses on the type of facial expression of emotion while attunement focuses on how mother and twin infant change their valence of facial expression of emotions. Emotional completement was coded when partner (2) expressed the positive valence of facial expression of emotion of partner (1). Thus, for example, emotional completement was coded when the mother expressed pleasure while the infant was interested in the mother.

Continued microanalysis of non-stable emotional expressions entailed to a combination of dyadic emotional expressions in the course of the same subunit of analysis. For example, one subunit started with an emotional matching which was followed by a completement and ended in a mismatching. Alternatively, one subunit started with a mismatching that was followed by a matching, and so on.

One feature that comes out from the temporal organization of nonstable dyadic emotional expressions is the direction, that is, which partner matched first, or completed first the emotional state which had been expressed by the other partner, or which partner was the first to cause mismatching. It has to be noted that when the beginning of an emotional matching/completement/mismatching preceded the beginning of the subunit of analysis, the direction of it was not coded.

Data micro-analysis was carried out by the first author of this paper (M.M.). The first author was trained by the second author (T.K.) who has constructed the coding system and has a long experience on micro-analysis. Training was carried out according to the following 
Citation: Markodimitraki M, Kokkinaki T (2015) Exploring Emotional 'Dialogues' in Spontaneous Twin Infant-mother Interaction. Int J Psychol Behav Anal 1: 101. doi: http://dx.doi.org/10.15344/2455-3867/2015/101

plan: a) study of the literature on the theoretical background of this study with special emphasis on emotional expressions; b) instruction on the operational definitions of the type and the valence of facial expression of emotions and the interpersonal engagement categories. Then, the first author was trained on the correspondence between these operational definitions and images of infant and mothers' facial expressions in the video-recordings; and c) repeated independent micro-analysis by the first researcher followed by corrections and discussions with the second researcher. The same plan was followed for the training of the student who participated in the inter-observer reliability testing though she was not aware of the hypotheses under investigation of this study.

\section{Inter-observer reliability}

Inter-observer reliability assessments of a random sample of 33\% of video-files were made for the type, the valence and the intensity of twin infant and maternal facial expressions of emotion. Inter-observer reliability for all categories ranged from 0.77 to 0.87 , the mean value of $\mathrm{k}$ (Cohen's kappa) being 0.81 . In particular, inter-observer reliability for the type, the valence and the intensity of facial expressions of emotions ranged from 0.77 to $0.84,0.79$ to 0.87 , and from 0.77 to 0.85 , respectively. The increased task demands on the observer and the multiple messages of a complex interaction itself are recognized as two factors that contribute to the difficulty of establishing high interobserver reliability [36]. Kappas over 0.75 has been characterized as excellent [37]. After the end of inter-observer reliability assessments, the two scorers discussed and corrected each assessment on which there was disagreement. The statistical analysis was carried out after all the corrections were made on the data set.

\section{Statistical analysis}

This experiment, by its nature, generated data with relatively strong dependencies between them since repeated observations on time are obtained on a relatively small number of individuals. These longitudinal dependencies were not taken into account in the analysis because of their complexity. Therefore, for the statistical analysis, the chi-square test of independence was used to determine possible relationships between pairs of categorical variables. This would tend to increase the number of significant results. For this reason, the significance level was set at $1 \%$ (instead of $5 \%$ ), as a safeguard against false rejections of the null hypothesis, increasing therefore the threshold of the analysis. In the case of a significant result, adjusted residuals were calculated to indicate categories that deviate from the independence assumption. Adjusted residuals follow approximately a standard normal distribution if the independence assumption is correct [38]. Therefore, in the following results, conclusions of a significant result are reached only if a combination of categories has an adjusted residual whose absolute value is greater than 2.0 , since this combination seems to deviate from the independence assumption. Friedman test (with sliding windows) was used to explore possible age-related changes of emotional expressions across the five data points. Through Friedman analysis, we took into consideration the fact that we had different number of subunits for each subject and at each age point. The significance level for Friedman test set at 5\%.

\section{Results}

In the course of 203 minutes of spontaneous twin infant-mother interaction, maternal infant-directed speech was segmented into 2,571 subunits within 683 units of analysis.

\section{Hypothesis $1 a$ and $1 b$ : Frequency and rate of occurrence of emotional coordination and mismatching}

Out of the total 2,571 subunits, infant and maternal emotional expressions remained stable in 2,178 (84.7\%) subunits. Within subunits of stable emotional states, emotional mismatching (1017, $46.7 \%)$ predominated over matchings $(906,41.6 \%)$ and completement $(255,11.7 \%)$. Within the total number of subunits, infant and maternal emotional expressions were not stable in 393 (15.3\%) subunits. The combinations of matching and mismatching (135, 34.4\%) predominated over the combinations of matching and completement (108, 27.5\%), matching, completement and mismatching (46, $11.7 \%)$, completement and mismatching $(25,6.4 \%)$, or intermittent mismatching $(58,14.8 \%)$, matching $(20,5.1 \%)$, or completement (1, $0.3 \%)$.

A total of 1,344 emotional matching, 470 completements and 1,423 mismatching occurred in the course of 203 minutes of free mother-infant interaction, giving an average of 6.6 matchings, 2.3 completements and 7.0 mismatchings per minute, respectively.

\section{Hypothesis 1a: Relationship between twin infant and maternal emotional expressions}

A relationship between twin infant and maternal pleasure, interest and neutral emotion $\left(\chi^{2}=433.937, \mathrm{df}=4, \mathrm{p}<0.001\right)$ (see Note 2) showed that when the mother was expressing pleasure, the infant was also pleased (53.9\%) (pleasure matching) more than when the mother was showing interest (15.7\%), or was neutral in emotion (9.4\%). When the mother was showing interest, the infant was more likely to be interested (47.9\%) (interest matching) than when the mother was pleased $(21.5 \%)$, or was neutral in emotion $(15.8 \%)$. When the mother was neutral in emotion, the infant was more likely to be also neutral (74.8\%) (neutral matching) than when the mother was pleased (24.5\%), or interested (36.3\%).

Further, a relationship between the mothers' pleasure, interest and neutral emotion and the infants' interest to the external world and negative emotion $\left(\chi^{2}=22.959, \mathrm{df}=2, \mathrm{p}<0.001\right)$ showed that when the mother was expressing pleasure, or interest to the infant, the infant was more likely to be interested in the external world $(81.4 \%$ and $79.5 \%$ ) (emotional mismatching) than when the mother was neutral in emotion (60.1\%). When the mother was neutral in emotion, the infant was more likely to express negative emotion (39.9\%) (mismatching) than when the mother was pleased (18.6\%), or interested to the infant (20.5\%).

\section{Hypothesis 1a: Relationship between twin infant and maternal emotional intensity}

A relationship between infant and maternal emotional intensity $\left(\chi^{2}=\right.$ $842.017, \mathrm{df}=9, \mathrm{p}<0.001)$ provided evidence that when the emotions of the mother were ascending, descending, stable or fluctuating, the twin infant's emotions changed rather in the same (ascending, descending, stable or fluctuating, respectively) (attunement) than a different direction.

\section{Hypothesis 2: Direction of emotional coordination and mismatching}

In the course of non-stable infant and maternal emotional expressions, infants matched [130 (56\%) vs 103 (44\%), Binomial test, $\mathrm{p}=0.08]$, completed maternal emotional expressions [82 (53\%) vs 73 (47\%), Binomial test, $\mathrm{p}=0.52$ ], or caused mismatching [ $156(61.6 \%)$ vs $97(39.4 \%)$, Binomial test, $\mathrm{p}<0.001]$ more than vice versa, though the 
Citation: Markodimitraki M, Kokkinaki T (2015) Exploring Emotional 'Dialogues' in Spontaneous Twin Infant-mother Interaction. Int J Psychol Behav Anal 1: 101. doi: http://dx.doi.org/10.15344/2455-3867/2015/101

difference was significant only for mismatching.

Hypothesis 3: Relationship of twin infant and maternal facial expressions of emotion between the beginning and the end of maternal infant-directed speech

A relationship between maternal emotions in the beginning and at the end of spontaneous maternal speech $\left(\chi^{2}=5581.589, \mathrm{df}=9, \mathrm{p}<0.001\right)$ (see Note 3 ) shows that when the mother was pleased, interested to the partner, or to the external world, or neutral in emotion in the beginning of maternal speech, it was more highly probable to remain in the same emotional state, than change, at the end of it. Similar emotional consistency was evidenced for twin infant emotions in the beginning and at the end of maternal speech $\left(\chi^{2}=7780.594, \mathrm{df}=16\right.$, $\mathrm{p}<0.001)$.

Hypothesis 4: Infant age effect on maternal and twin infant emotions and on emotional matching, completement and mismatching

Friedman test analysis provided evidence of non-significant changes of maternal and infant emotions and emotional matching, completement and mismatching across the 5 age points of this study. Figure 1 shows the similar but non-linear developmental curves of emotional matching, completement and mismatching.

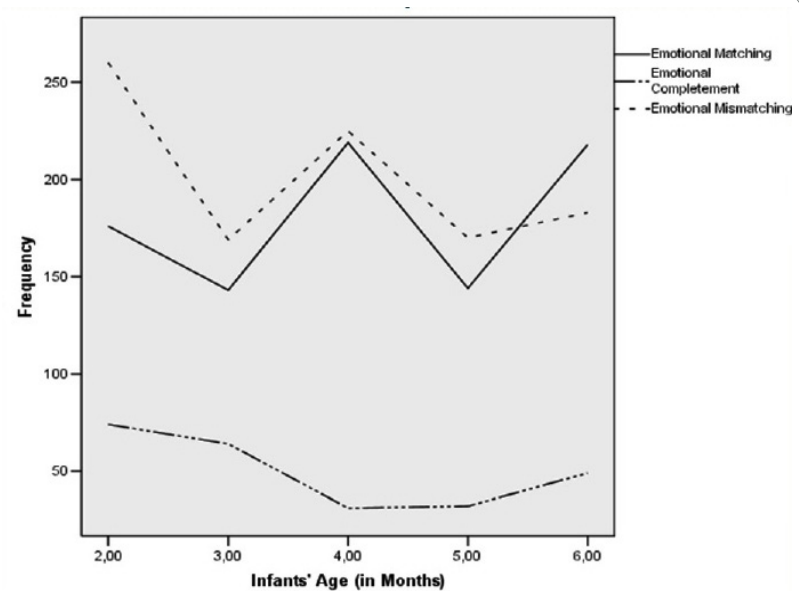

Figure 1: Developmental Trajectories of Emotional Matching, Completement and Mismatching in Spontaneous Twin InfantMother Interaction.

\section{Discussion}

We aimed to investigate emotional coordination and mismatching in the course of spontaneous twin infant-mother interaction from the $2^{\text {nd }}$ to the $6^{\text {th }}$ month of infants' life. We provided evidence that: a) emotional coordination along with mismatching takes place in spontaneous twin infant-mother interaction. Emotional matching and mismatching occurred at similar rates; b) infants directed emotional coordination and caused mismatching more than mothers did, though the difference was significant only for mismatching c) the emotional states of twin infants and their mothers remained consistent in the beginning and at the end of maternal infant-directed speech; and d) the developmental non-linear trajectories of emotional matching, completement and mismatching along with maternal and infant emotions did not change significantly across the age range of 2-6 months. These results verify part of the first hypothesis, the second and the third hypothesis and confirm, directly and indirectly, the results of previous relevant studies $[9-11,15,25]$

Inter-subjective emotional coordination along with intra-subjective emotional coherence in spontaneous twin infant-mother interaction reveal coordination within - and between - twin infants and mothers in three essential dimensions of communication that motivate learning in a human community: 'kinematics' (the temporal patterns of movements), 'physiognomics' (spatial patterns or forms) and 'energetics' (force or effort) [24]. Timing adjustment in twin infant-mother interaction is presupposed by the way both partners temporally structure and synchronize their facial expressions. In particular, in this study emotional coordination presupposes that one partner (2) expressed: a) the type (matching), b) the positive valence (completement), and c) the shifts of emotional intensity (attunement) of the other partner (1), within frames of a second, before or after, the first partner's emotional expressions. These support the assumption that twin infants not only are they able to adjust or fit their emotions to the emotions of their mothers, but they participate actively and control to a great extent the exchange $[21,22,39]$. This gains further support from the evidence that, infants directed more than mothers mismatching, matching and completement.

We assume that form adjustment at three interrelated levels- the muscular, the neural and the somatic/autonomic- is revealed indirectly by the matching of facial expressions of emotions in twin infant-mother interaction. At the muscular level, form adjustment is evidenced by emotional matching according to the definitions of the type of facial expressions of emotions [33]. At the neural level, support comes from the discovery of 'mirror neurons' (MNs), a group of neurons that has the capacity to discharge both when performing an action and when observing the same action performed by another. At the somatic/autonomic level, support comes from the assumption that the sharing of impulses to move, and the feelings associated with them, engage motor images in reciprocal and emotionally charged negotiations of possible cooperative activity [40].

Energy adjustment is evidenced by emotional attunement of facial expressions of emotions, as implied by the matching in the direction of emotional shifts. This provides evidence that not only singletons but also twin infants can experience both self-awareness and otherawareness as well as awareness of purposeful sharing of emotional states $[21,22]$. This suggestion is reinforced by the intra-subjective emotional coherence taking place in the course of spontaneous maternal twin infant-directed speech. This kind of consistency suggests that both twin infants and their mothers express intra-coordination, that is, coherence in awareness, consciousness and purpose, unified intentionality and control of internal states $[22,24,41]$.

Further, in this study we evidenced similar rates of emotional matching and mismatching, along with combinations of: a) matching with mismatching; and b) matching with completement in spontaneous twin infant-mother interaction. These patterns are integrated in the assumption that intersubjective communication requires the infant to interact with the other in a matching and self-coordinated activelyattuned way. At the same time, the expressed states of feelings in either one of the partners are evidently incomplete or open, anticipating a particular range of possible reactions of feelings in the expressions of the partner [22]. To perceive another person and respond appropriately, the infant must be capable of integrating from perception of the other's face movements, an awareness of, or sympathy with, the generative emotion behind these expressions. This requires a specific readiness 
Citation: Markodimitraki M, Kokkinaki T (2015) Exploring Emotional `Dialogues' in Spontaneous Twin Infant-mother Interaction. Int J Psychol Behav Anal 1: 101. doi: http://dx.doi.org/10.15344/2455-3867/2015/101

to grasping the other's behavior, discriminating and differentially responding to the different emotions behind the expressions (thereby to perceive their motives) and to the changing value of motives $[21,22]$. Further, the evidence of transitions between emotional coordination and mismatching is integrated in "emotional narratives", the fundamental carriers of information about motivational changes in the short term [21]. The motive impulses of human subjects are adapted to pass readily between them. The close following of emotions between persons in direct and intimate communication is adapted to intersubjective functions, that is, the coordination of cognitive dynamics, attention shifts, changes in motive force in each self and between selves $[21,22,42]$. This study provides evidence that the sharing of feelings (matching) and climaxes of intensity (attunement) may be impossible unless chaining and grouping of emotional behaviors are brought into close harmony by smooth continuation (completement), or strategic interruption, expressed through inappropriateness in forms of emotions (mismatching). Interruption signifies that the towards a different physiognomy indicative of agitation, distress and need for attention to some internal physiological need, or for comfort. We assume that periods of emotional mismatching constitute "renegotiations" of intentions, or subtle varieties of motive adjustment in the course of which the brains of both participants (twin infant and mother) still continue to be re-organized.

Our conclusions are limited in a number of ways. First, the obvious limitation of this study is the small Greek, Cretan sample size and more research is necessary to determine whether our findings will be replicated to other populations (see Introduction). Second, in this study the focus was on facial expressions, as index of emotions and this has to be completed by the investigation of other expressive systems that convey affective information [43]. Third, the twin ecology of firstborn and second-born twin infants has to be compared, along with the interaction context of singletons. Based on these limitations, we view our findings as preliminary until micro-analysis and comparisons of emotional expressions of a large sample of twin infant- and singletonmother dyads are undertaken.

In sum, this study extends empirically the theory of innate intersubjectivity to twin infant-mother interaction and provided evidence that both mothers and twin infants adjust the timing, form and energy of their emotional expressions in order to obtain intersynchrony (through matching and attunement), transitions of intermotive adjustments (through mismatching) and complementarity of feelings (through completement).

\section{Notes}

1. The term "completement" has been selected mainly for two reasons: a) interest has been taken to be the most frequently experienced positive emotion [44], and b) the expressed states of feeling in either one of the interacting partners are evidently incomplete or open, anticipating a particular range of possible reactions of feeling in the expression of the partner [22].

2. In order to avoid low frequency cells in the analysis of the relationship between twin infant and maternal emotions, two analyses were carried out, one for the relationship between twin infant and maternal pleasure, interest and neutral emotion, and one for the relationship between maternal pleasure, interest and neutral emotion and twin infant's interest to the external world and negative emotion. Each of these analyses was based on the motivation for communication changes and its expressions change

construction of variables according to which the expression of an emotion was stable and exclusive. This implies that all the cases in which one emotion was preceded or followed by another emotion were excluded from this analysis.

3. Analysis of the relationship of each partner's emotions in the beginning and at the end of maternal speech included all maternal and infant emotions with the exception of maternal negative emotion which was excluded due to low frequencies.

\section{Competing Interests}

The authors declare that they have no competing interests.

\section{Author's contribution}

Both authors contributed substantially to conception and design, acquisition and analysis of data and interpretation of results.

\section{Acknowledegements}

We are deeply indebted to the infants and their families for offering their time, cooperation and patience to participate in the study.

\section{References}

1. Muir D, Lee K, Hains C, Hains S (2005) Infant perception and production of emotions during face-to face interactions with live and "virtual" adults. In: Nadel J \& Muir D (Eds) Emotional development: Recent research advances, Oxford University Press 207-233.

2. Bråten S, Trevarthen C (2007) Prologue: From infant inter subjectivity and participant movements to simulation and conversation in cultural common sense. In: Bråten S (Ed) On Being Moved: From Mirror Neurons to Empathy, John Benjamins 21-34.

3. Kaye K \& Fogel A (1980) The temporal structure of face-to-face communication between mothers and their infants. Developmental Psychology 16: 454-464.

4. Tronick EZ, Cohn JF (1989) Infant-mother face-to-face Interaction: Age and gender differences in coordination and the occurrence of miscoordination. Child Dev 60: 85-92.

5. Weinberg M K, Tronick EZ, Cohn J F, Olson KL (1999) Gender differences in emotional expressivity and self-regulation during early infancy. Deve Psychol 35: 175-188.

6. Stern D, Gibbon J (1978) Temporal expectancies of social behaviours in mother-infant play. In: Thoman E (Ed) Origins of the infant's social responsiveness, Erlbaum, New York 409-429.

7. Fogel A (1977) Temporal organization in mother-infant face-to-face interaction. In: Schaffer H R (Ed) Studies in mother-infant interaction: The Loch Lomond Symposium, Academic Press, London, 119-152.

8. Cassidy KW, Fineberg DS, Brown K, Perkins A (2005) Theory of mind may be contagious, but don't catch it from your twin. Child Dev 76: 97-106.

9. Feldman R, Eidelman A (2004) Parent-infant synchrony and the socialemotional development of triplets. Dev Psychol 40: 1133-1147.

10. Feldman R, Eidelman Al (2005) Does a triplet birth pose a special risk for infant development? Assessing cognitive development in relation to intrauterine growth and mother-infant interaction across the first 2 years. Pediatrics 115: 443-452.

11. Feldman R, Eidelman Al, Rotenberg N (2004) Parenting stress, infant emotion regulation, maternal sensitivity, and the cognitive development of triplets: A model for parent and child influences in a unique ecology. Child Dev 75: 1774-1791.

12. Szajnberg NM, Skrinjaric J, Moore A (1989) Affect attunement, attachment, temperament, and zygosity: A twin study. J Am Acad Child Adolesc Psychiatry 28: 249-253.

13. Zahn-Waxler C, Robinson JL, Emde RN (1992) The development of empathy in twins. Dev Psychol 28: 1038-1049.

Int J Psychol Behav Anal

ISSN: 2455-3867

IJPBA, an open access journal Volume 1(1). 2015. 101 
Citation: Markodimitraki M, Kokkinaki T (2015) Exploring Emotional 'Dialogues' in Spontaneous Twin Infant-mother Interaction. Int J Psychol Behav Anal 1: 101. doi: http://dx.doi.org/10.15344/2455-3867/2015/101

14. Caspi A, Moffitt TE, Morgan J, Rutter M, Taylor A \& et al. (2004) Maternal expressed emotion predicts children's antisocial behavior problems: Using monozygotic-twin differences to identify environmental effects on behavioral development. Dev Psychol 40: 149-161.

15. Trevarthen C, Daniel S (2005) Disoranized rhythm and synchrony: Early signs of autism and Rett syndrome. Brain Dev 27: S25-S34.

16. Beebe B (1982) Micro-timing in mother-infant communication. In Kaye M R (Ed) Non-verbal communication today, The Hague: Mouton. 169-195.

17. Losoya SH, Callor S, Rowe DC, Goldsmith HH (1997) Origins of familial similarity in parenting: A study of twins and adoptive siblings. De Psychol 33: 1012-1023.

18. Terkenli TS, Bellas ML, Jenkins LD (2007) Tourism impacts on local life: Socio-cultural continuity and change in Crete. Aegean Geograph J 16: $37-$ 52.

19. Keller H, Zach $\cup(2002)$ Gender and birth order as determinants of parental behavior. Int Jof Behav Dev 26: 177-184.

20. Trevarthen C (1977) Descriptive studies in infant behavior. In: Schaffer H $\mathrm{R}$ (Ed.), Studies in mother-infant interaction. Academic Press, London, 27270

21. Trevarthen C (1993a) The self born in inter subjectivity: the psychology of an infant communicating. In: Neisser U (Ed.), The perceived self: ecological and interpersonal sources of the self-knowledge, Cambridge University Press 121-173.

22. Trevarthen $C(1993 b)$ The function of emotions in early infant communication and development. In: Nadel J \& Camaioni L (Eds), New perspectives in early communicative development, London: Routledge, London 48-81.

23. Trevarthen C (2005) Action and emotion in development of cultural intelligence: why infants have feelings like ours. In: Nadel J \& Muir D (Eds) Emotional development: Recent research advances, Oxford University Press 61-91.

24. Trevarthen C (1986) Development of intersubjective motor control in infants. In: Wade M G \& Whiting H T A (Eds) Motor development in children: Aspects of coordination and control. 209-261.

25. Markodimitraki M (2003) The psychology of twins: Imitation and emotion in a pair of heterozygous twins.

26. Lytton H, Watts D, Dunn B (1987) Twin-singleton differences in verbal ability: Where do they stem from? Intelligence 11: 359-369.

27. Feldman R (2007) Parent-infant synchrony: Biological foundations and developmental outcomes. Current Directions in Psychological Science 16 340-345.

28. Hulshoff Pol HE, Posthuma D, Baare WF, De Geus EJ, Schnack HG, et al. (2002) Twin-singleton differences in brain structure using structura equation modelling. Brain 125: 384-390.

29. Hollingshead AB (1975) Four Factor Index of Social Status. Unpublished manuscript. Department of Sociology, Yale University, New Haven.

30. Wilson RS (1983) The Louisville twin study: Developmental synchronies in behavior. Child Dev 54: 298-316.

31. Goldsmith HH (1991) A zygosity questionnaire for young twins: A research note. Behav Genet 21: 257-269.

32. Pateraki M, Markodimitraki M, Kaklamani S, Kugiumutzakis G (2008) Imitative direction and kinds in DZ twin infant pair interactions 14: 65-90.

33. Kokkinaki T (2009) Emotional expressions during early infant - father conversation. European J Dev Psychol 6: 705-721.

34. Kokkinaki T (2011) Coding system for the microanalysis of parental speech in spontaneous infant-parent imitation. Retrieved from PsycTESTS.

35. Kokkinaki T, Vasdekis VGS (2008) Gender as a determinant of emotional expressions in spontaneous infant-father interactions: an exploratory study. In Glenyn B C \& Zini R P (Eds) New Directions in Developmenta Psychobiology, Nova Science Publications 117-150.
36. Bakeman R, Gottman JM (1986) Observing Interaction: An Introduction to Sequential Analysis. Cambridge University Press.

37. Fleiss JL (1981) Statistical methods for rates and proportions. Wiley

38. Dobson AJ (2001) An introduction to generalized linear models. London: Chapman and Hall.

39. Kugiumutzakis G (1993) Inter subjective vocal imitation in early mother-infant interaction. In: New perspectives in early communicative development, Routledge 23-47.

40. Trevarthen C (2009) Human biochronology: on the source and function of musicality. 221-265

41. Izard CE (1992) Basic emotions, relations among emotions and emotioncognition relations. Psychol Rev 99: 561-565

42. Trevarthen C (1997) The nature of motives for human consciousness, Psychology: the Journal of the Hellenic Psychological Society 4: 187-221.

43. Weinberg MK, Tronick EZ (1994) Beyond the face: an empirical study of infant affective configurations of facial, vocal, gestural, and regulatory behaviors. Child Dev 65: 1503-1515.

44. Izard CE and Buechler S (1980) Aspects of consciousness and personality in terms of differential emotions theory. In: Plutchik R \& Kellerman H (Eds) Emotion: Theory, research and experience. Academic Press 165-187. 